\title{
Nonlinear Integral Sliding Mode Control for a Second Order Nonlinear System
}

\author{
Xie Zheng, Xie Jian, Du Wenzheng, and Cheng Hongjie \\ Xian High Technology Research Institute, Xian 710025, China \\ Correspondence should be addressed to Du Wenzheng; 15332439207@163.com
}

Received 23 November 2014; Accepted 7 January 2015

Academic Editor: Onur Toker

Copyright (C) 2015 Xie Zheng et al. This is an open access article distributed under the Creative Commons Attribution License, which permits unrestricted use, distribution, and reproduction in any medium, provided the original work is properly cited.

\begin{abstract}
A nonlinear integral sliding-mode control (NISMC) scheme is proposed for second order nonlinear systems. The new control scheme is characterized by a nonlinear integral sliding manifold which inherits the desired properties of the integral sliding manifold, such as robustness to system external disturbance. In particular, compared with four kinds of sliding mode control (SMC), the proposed control scheme is able to provide better transient performances. Furthermore, the proposed scheme ensures the zero steady-state error in the presence of a constant disturbance or an asymptotically constant disturbance is proved by Lyapunov stability theory and LaSalle invariance principle. Finally, both the theoretical analysis and simulation examples demonstrate the validity of the proposed scheme.
\end{abstract}

\section{Introduction}

Control strategies design for nonlinear systems has attracted considerable research interest in the recent past [1-4]. Sliding mode control (SMC) as an effective robust control scheme has been successfully applied to a wide variety of systems $[5,6]$. In spite of claimed robustness, the SMC suffers certain drawbacks, mainly the chattering phenomenon [7, 8]. This phenomenon is extremely harmful to the actuators of physical systems [9]. To prevent chattering in the SMC, Slotine proposed quasi-SMC which introduced the bounded layer in SMC [10]. In [11], another scheme is based on the observer design which suppresses the high frequency oscillations of the control input. Though the chattering phenomenon could be attenuated by above-mentioned approaches, the approaches would bring in large steady-state error in the presence of the nonlinear system with disturbances. In [12], Chern and Wu first proposed integral sliding mode control (ISMC) which significantly enhanced the robustness against external disturbance of the nonlinear system.

The disturbance and uncertainty can be effectively restrained by ISMC; nevertheless, the control performance (overshoot and respond time) would become very poor if the initial errors of the system are very large. Much worse, duo to the effect of "integral windup" phenomenon, the ISMC system maybe unstable in the presence of control signal control input constraints [13-15]. To alleviate the above shortcomings, Cho et al. proposed a small gain ISMC, but the small gain got the response speed of the system slow [16]. In [17], Lee proposed global integral sliding mode control, this approach achieved that all the states locate in the sliding mode manifold at the beginning and keeps this stage to eliminate the reaching stage.

In this work, aiming at improving control performance for second order systems, a nonlinear ISMC scheme was proposed. Firstly, a nonlinear integral sliding manifold with saturated function was designed, which could eliminate the effect of system initial error. Secondly, based on the proposed sliding manifold, the sliding mode control law design was carried out in two parts: (1) case of control input without constraints and (2) case of control input constraints. In addition, by the Lyapunov stability theory and LaSalle invariance principle, we have proved that the proposed scheme ensured the zero steady-state error in the presence of a constant disturbance or an asymptotically constant disturbance. Furthermore, through theory analysis, the research proved that the proposed control scheme could be equivalent to PD controller with nonlinear integral control 
scheme. Finally, a numerical example has been provided to demonstrate the effectiveness of the obtained results.

\section{Problem Statement}

Consider a second order nonlinear system described by the state equation:

$$
\begin{gathered}
\dot{x}_{1}=x_{2} \\
\dot{x}_{2}=f(\mathbf{x}, t)+u(t)+d(t) \\
y=x_{1},
\end{gathered}
$$

where $x_{1}, x_{2}$ are the state variables of the system and $\mathbf{x}=$ $\left[x_{1}, x_{2}\right] \in R^{2}$ is the state vector, $f(\mathbf{x}, t)$ is a sufficiently smooth vector field, $u(t)$ denotes the input control signal, and $d(t)$ denotes external disturbances. For simplicity, it is assumed.

Assumption 1. $f(\mathbf{x}, t)$ is a bounded function with uncertainty, and $f(\mathbf{x}, t)$ consists of nominal parts $\widehat{f}(\mathbf{x}, t)$ which are known a priori and uncertain parts $\tilde{f}(\mathbf{x}, t)$ which are bounded and unknown [18]. Furthermore, $\widetilde{f}(\mathbf{x}, t)$ satisfy

$$
\begin{gathered}
\tilde{f}(\mathbf{x}, t)=f(\mathbf{x}, t)-\widehat{f}(\mathbf{x}, t) \\
|\widetilde{f}(\mathbf{x}, t)| \leq F(\mathbf{x}, t) .
\end{gathered}
$$

Assumption 2. $d(t)$ denotes external disturbances of the system, which satisfy

$$
|d(t)| \leq D(t) .
$$

Define the tracking error as

$$
e=y-y_{d}
$$

where $y_{d}$ denotes the reference signal. The role of the controller is to ensure that system output accurately track the reference signal.

\section{Nonlinear Integral Sliding Mode Manifold Design}

When the system is perturbed or uncertain, the finite time stabilization is not ensured. Hence, in this section a reaching law based discontinuous control law is developed which rejects the uncertainties of the system and ensures that the control objectives are fulfilled.

For system (1), if using the traditional sliding mode control scheme, the sliding manifold can be described by the following equation:

$$
S=\dot{e}+c e,
$$

where $c$ is a strictly positive constant.

To reduce the steady-state error, an integral term of tracking error is introduced into (6), which makes up the traditional integral sliding manifold:

$$
S=\dot{e}+c e+c_{1} \int_{0}^{t} e d \tau,
$$

where $c_{1}$ is a strictly positive constant.
To enhance the robustness of closed loop system, a sliding manifold design incorporates global integral sliding manifold which results in the elimination of reaching phase [19]. The global integral sliding manifold can be described as follows:

$$
S=\dot{e}+c e+c_{1} \int_{0}^{t} e d \tau-\dot{e}(0)-c e(0) .
$$

A nonlinear integral sliding manifold is proposed in this research. With the integral manifold is given as

$$
\begin{gathered}
S=\dot{e}+c e+c_{1} \zeta \\
\dot{\zeta}=\sigma(e),
\end{gathered}
$$

where $\sigma(e)$ is a new nonlinear saturation function which enhances small errors and will be saturated with large errors in shaping the tracking errors. In order to research the properties of $\sigma(e)$, a potential energy function $\Phi(e)$ is introduced as

$$
\Phi(e)= \begin{cases}\frac{2 \beta^{2}}{\pi}\left(1-\cos \frac{\pi e}{2 \beta}\right), & |e|<\beta \\ \beta e-\frac{\pi-2}{\pi}, & e>\beta \\ -\beta e-\frac{\pi-2}{\pi} \beta^{2}, & e<-\beta,\end{cases}
$$

where $\beta$ is the design parameter of $\Phi(e)$. The time derivative of (8) along e can be written as

$$
\sigma(e)= \begin{cases}\beta \sin \left(\frac{\pi e}{2 \beta}\right), & |e|<\beta \\ \beta, & e>\beta \\ -\beta, & e<-\beta .\end{cases}
$$

There is the following lemma for (9) and (10).

Lemma 3 (see [13]). $\Phi(e)$ and $\sigma(e)$ satisfy the following:

(1) $\Phi(e)>0$ for $e \neq 0, \Phi(e)=0$ for $e=0$;

(2) $\Phi(e)$ is a second order continuous differentiable function. $\sigma(e)$ is a strictly monotone increasing function for $|e|<\beta$, and $\sigma(e)$ is a saturated function for $|e| \geq \beta$.

The above properties of $\Phi(e)$ and $\sigma(e)$ can be obtained by some simple mathematical operations.

Figure 1 shows the curves of the potential function $\Phi(e)$, $\sigma(e)$, and $e(\beta=1)$. It is obvious from Figure 1 that the proposed integral sliding manifold enhances small errors and will be saturated with large errors in shaping the tracking errors. Namely, when $|e| \leq \beta,|\sigma(e)| \geq e$; when $|e|>$ $\beta,|\sigma(e)|= \pm \beta<e$. Furthermore, the desired control performance would be obtained by choosing a proper design parameter $\beta$.

\section{Sliding Mode Control Law Design}

Base on the proposed integral sliding manifold, the sliding mode control law design is carried out in two parts: (1) case of control input without constraints and (2) case of control input constraints. 


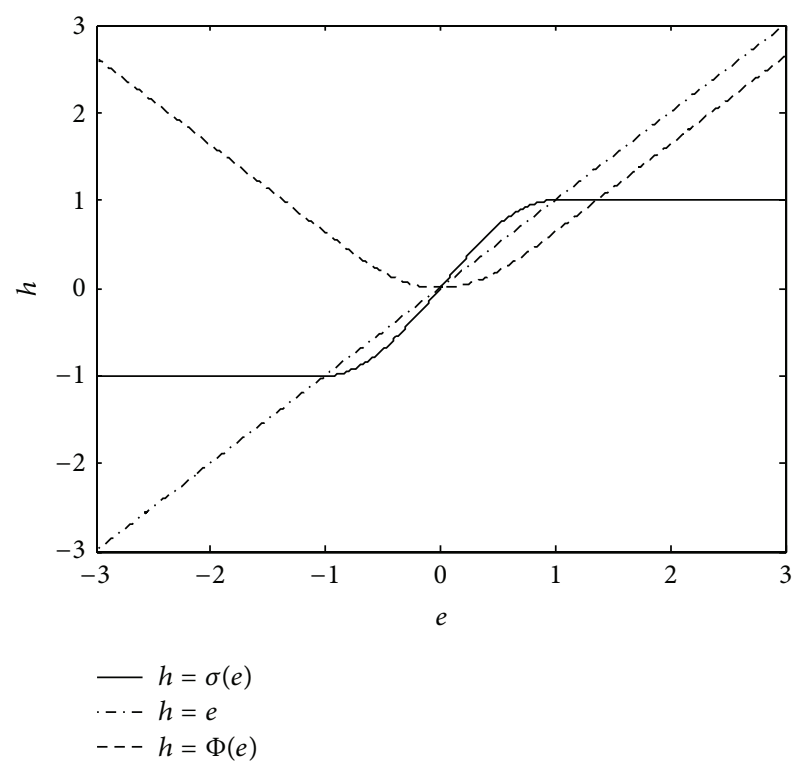

FIgURE 1: The curves of $\Phi(e), \sigma(e)$, and $e(\beta=1)$.

4.1. Case of Control Input without Constraints. According to the reaching law condition $S \dot{S} \leq-\eta|S|, \eta>0$, and use the nonlinear saturation function $\operatorname{sat}(\bullet)$ to smoothen the input control signal $u$. Hence, the control law of control input without constraints can be obtained as

$$
u(t)=\widehat{u}-k(\mathbf{x}, t) \operatorname{sat}\left(\frac{S}{\delta}\right)
$$

where $\delta$ is a constant that denotes the thickness of the boundary layer, $\widehat{u}$ denotes nominal input control signal, and $k(\mathbf{x}, t)$ denotes switching gain.

Theorem 4. System (1) combined with the control law described by (11) assures the stabilization of the sliding manifold defined by (8). Meanwhile, the sliding manifold can reach the boundary layer within finite time.

Proof. When the sliding manifold $s$ is outside of boundary layer, (11) can be rewritten as

$$
u(t)=\widehat{u}-k(\mathbf{x}, t) \operatorname{sgn}(S)
$$

where $\operatorname{sgn}(\bullet)$ denotes the sign function. By taking the time derivative of (11), one has

$$
\dot{S}(t)=\ddot{e}+c \dot{e}+c_{1} \sigma(e) .
$$

The following Lyapunov candidate function is defined:

$$
\begin{gathered}
V_{1}=\frac{1}{2} S^{2}, \\
\dot{V}_{1}=S\left(\ddot{e}+c \dot{e}+c_{1} \sigma(e)\right) \\
=S\left(f(\mathbf{x}, t)+u+d(t)-\ddot{y}_{d}+c \dot{e}+c_{1} \sigma(e)\right) \\
=S(f(\mathbf{x}, t)+u-k(\mathbf{x}, t) \operatorname{sgn}(S)+d(t)-\ddot{y} d+c \dot{e} \\
\left.\quad+c_{1} \sigma(e)\right) \\
\leq|S|(|f(\mathbf{x}, t)+d(t)|)-(F(\mathbf{x}, t)+D(t)+\eta) \\
\leq-\eta|S| .
\end{gathered}
$$

Therefore, the sliding manifold $S$ is asymptotically stable. Meanwhile, sliding manifold $S$ can reach the boundary layer within finite time $t_{r}$ :

$$
t_{r} \leq \frac{|S(0)|-\delta}{\eta} .
$$

Definition 5. For simplicity, define

$$
\begin{aligned}
w(\mathbf{x}, t) & =\tilde{f}(\mathbf{x}, t)+d(t), \\
\gamma & =\frac{k(\mathbf{x}, t)}{\delta} .
\end{aligned}
$$

Theorem 6. System (1) with the input control signal is defined as (8) and the control laws are chosen as (11), and if $w(\mathbf{x}, t)$ is constant or convergent to constant at last $\left(\lim _{t \rightarrow \infty} w(\mathbf{x}, t)=l\right.$, $l$ is a constant), $\lim _{t \rightarrow \infty} e=0$.

Proof. Within the boundary layer $|S| \leq \delta$, the trajectory of sliding manifold can be described as

$$
\dot{S}(t)=\ddot{e}+c \dot{e}+c_{1} \sigma(e)=w(t)-\gamma S(t) .
$$

Considering $\dot{S}(t)=0$, (18) yields the following:

$$
S(s)=\frac{w(s)}{s+\gamma},
$$

where $s$ denotes Laplace operator. Based on final-value theorem, one gets

$$
\begin{aligned}
\lim _{t \rightarrow \infty} S(t) & =\lim _{s \rightarrow 0}\left(\frac{s w(s)}{s+\gamma}\right) \\
& =\lim _{s \rightarrow 0} \frac{1}{s+\gamma} \cdot \lim _{s \rightarrow 0} s w(s) \\
& =\lim _{s \rightarrow 0} \frac{1}{s+\gamma} \cdot \lim _{t \rightarrow \infty} w(t) \\
& =\frac{l}{\gamma} .
\end{aligned}
$$


Obviously, $\dot{S}(t)$ is a uniformly continuous function, since $t \rightarrow \infty$ use of Barbalat lemma results in $\dot{S}(t) \rightarrow 0$ that implies

$$
\lim _{t \rightarrow \infty}\left(\ddot{e}+c \dot{e}+c_{1} \sigma(e)\right)=0 .
$$

Let the Lyapunov function candidate be defined as

$$
V_{2}=\frac{1}{2} \dot{e}^{2}+c_{1} \Phi(e)
$$

Consider that $G(e)$ is the radial unbounded function, and $\Phi(0)=0$. Hence, (22) is a radial unbounded function positive definite function. By taking the time derivative of (22) along (21), one has

$$
V_{2}=\ddot{e} \ddot{e}+c_{1} \sigma(e) \dot{e}=\dot{e}\left(-c \dot{e}-c_{1} \sigma(e)\right)+c_{1} \sigma(e) \dot{e}=-c \dot{e}^{2} \leq 0,
$$

where $\dot{V}_{2} \equiv 0$ imply $\dot{e} \equiv 0$. Based on LaSalle invariance principle, $(e=0, \dot{e}=0)$ is the globally asymptotically stable equilibrium point; that is, $\lim _{t \rightarrow \infty} e(t)=\lim _{t \rightarrow \infty} \dot{e}(t)=$ 0 .

Remark 7. Inspired by [13], if $\lim _{t \rightarrow \infty} w(\mathbf{x}, t)=l$, when using (5) as the sliding manifold, within the boundary layer $\dot{S}(t)=$ $\ddot{e}+c \dot{e}=w(t)-\gamma S(t)$; that is, $\lim _{t \rightarrow \infty} e(t)=l / c \gamma$. When taking (6) or (7) as sliding manifold, $\lim _{t \rightarrow \infty} e(t)=0$. From Theorem 6, the proposed nonlinear integral sliding mode control scheme not only retains the advantages of integral sliding mode but also eliminates the constant disturbances.

4.2. Case of Control Input Constraints. From (11), it is easy to obtain that the nominal control signal depends on status of the system (1). Therefore, it is difficult to calculate the nominal control signal. Usually, the saturated control scheme is used under the condition of nominal control signal control input constraints. For system (1), considering the saturated control scheme,

$$
|u| \leq u_{\max }, \quad u_{\max }>0
$$

where $u_{\max }$ is the saturated value of input control signal. Hence, the saturated control law can be described as

$$
u=-u_{\max } \operatorname{sat}\left(\frac{S}{\delta}\right)
$$

Using the traditional sliding manifold equation (6), the control law equation (11) can be rewritten as

$$
\begin{array}{r}
u=-u_{\max } \operatorname{sat}\left(\frac{\left(\dot{e}+c e+c_{1} \int_{0}^{t} e d \tau\right)\left(u_{\max } / \delta\right)}{u_{\max }}\right) \\
=u_{\max } \operatorname{sat}\left(\left(\frac{u_{\max }}{\delta}(-\dot{e})+\frac{c u_{\max }}{\delta}(-e)\right.\right. \\
\left.\left.+\frac{c_{1} u_{\max }}{\delta} \int_{0}^{t}(-e) d \tau\right) \cdot\left(u_{\max }\right)^{-1}\right) .
\end{array}
$$

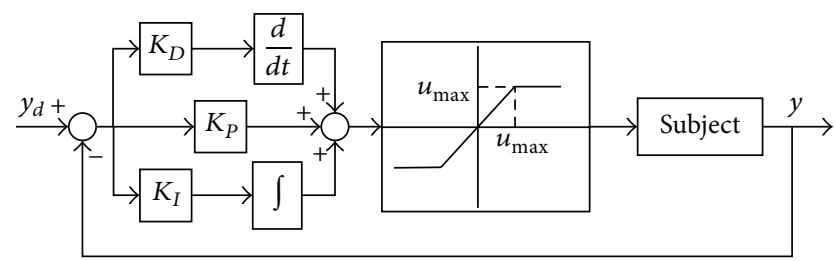

Figure 2: The schematic of the saturated controller (20) using sliding manifold equation (6).

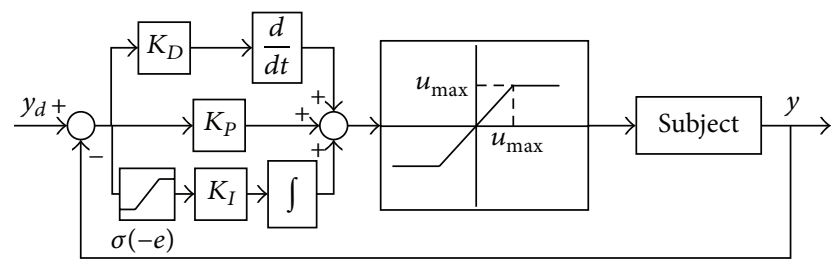

FIgURE 3: The schematic of the saturated controller using nonlinear sliding manifold equation (8).

Thus, the control law equation (26) can be equivalent to the traditional PID controller with saturated output, as shown in Figure 2. In Figure 2, $K_{P}=c u_{\max } / \delta, K_{I}=c_{1} u_{\max } / \delta$, and $K_{D}=c u_{\max } / \delta$.

Similarly, using the proposed sliding manifold equation (8), the control laws equation (11) can be obtained:

$$
\begin{array}{r}
u=-u_{\max } \operatorname{sat}\left(\frac{\left(\dot{e}+c e+c_{1} \int_{0}^{t} \sigma(e) d \tau\right)\left(u_{\max } / \delta\right)}{u_{\max }}\right) \\
=u_{\max } \operatorname{sat}\left(\left(\frac{u_{\max }}{\delta}(-\dot{e})+\frac{c u_{\max }}{\delta}(-e)\right.\right. \\
\left.\left.+\frac{c_{1} u_{\max }}{\delta} \int_{0}^{t}(-\sigma(e)) d \tau\right) \cdot\left(u_{\max }\right)^{-1}\right) .
\end{array}
$$

Meanwhile, the control law equation (27) can be equivalent to PD controller with nonlinear integral control scheme, as shown in Figure 3.

In Figure 3, the parameters $K_{P}, K_{I}$, and $K_{D}$ are same as those of Figure 2. The saturated value of input control signal is a constant. Therefore, the "windup" phenomenon can be eliminated by choosing a proper value of $\beta$.

\section{Examples}

This section presents a comparative study among controllers using traditional sliding manifold, integral sliding manifold, global integral sliding manifold, and nonlinear sliding manifold. Consider the following second order system given by

$$
\begin{gathered}
\dot{x}_{1}=x_{2} \\
\dot{x}_{2}=11 x_{1} x_{2}+(2+0.5 \sin (1.5 t)) x_{2}^{2}+10+u \\
y=x_{1},
\end{gathered}
$$




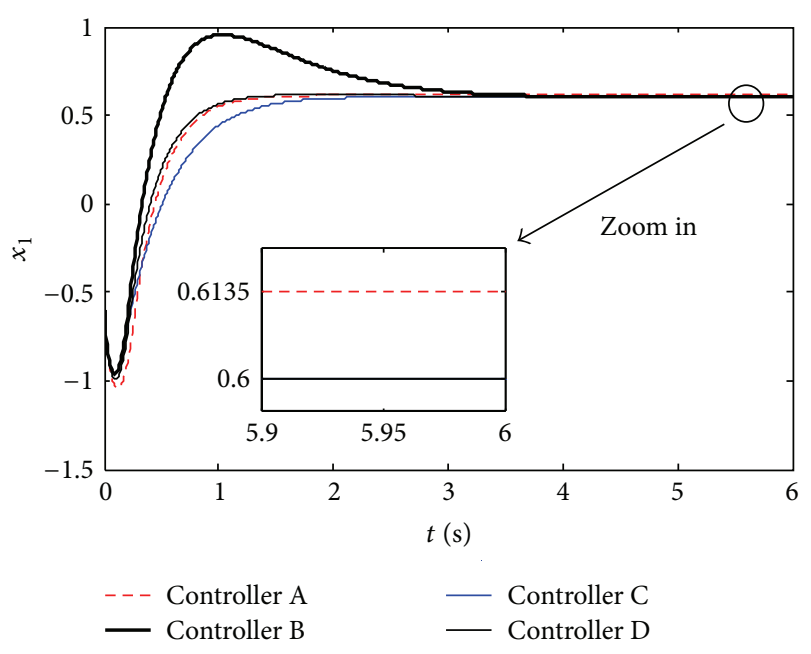

FIgURE 4: The response curves of state $x_{1}$.

where

$$
\begin{gathered}
f(\mathbf{x}, t)=11 x_{1} x_{2}+(2+0.5 \sin (1.5 t)) x_{2}^{2}, \\
\hat{f}(\mathbf{x}, t)=10 x_{1} x_{2}+2 x_{2}^{2}, \\
d=10, \\
|d| \leq D=11, \\
\tilde{f}(\mathbf{x}, t)=x_{1} x_{2}+0.5 \sin (1.5 t) x_{2}^{2}, \\
|\tilde{f}(\mathbf{x}, t)|=\left|x_{1} x_{2}+0.5 \sin (1.5 t) x_{2}^{2}\right| \leq\left|x_{1} x_{2}\right|+0.5 x_{2}^{2} \\
=F(\mathbf{x}) .
\end{gathered}
$$

The initial condition $x_{1}(0)=-0.6, x_{2}(0)=-8$, and the reference signal $y_{d}=0.6$. The switch gain $K=F+D+1=$ 33.3 , and boundary layer thickness $\delta=0.2$. The following are the four controllers:

controller A: using the traditional sliding manifold, the sliding manifold $S=\dot{e}+c e$;

controller B: using the integral sliding manifold, the sliding manifold $S=\dot{e}+4 e+4 \int_{0}^{t} e d \tau$;

controller C: using the global integral sliding manifold, the sliding manifold $S=\dot{e}+4 e+4 \int_{0}^{t} e d \tau-\dot{e}(0)-$ $4 e(0)$;

controller D: using the nonlinear sliding manifold, the sliding manifold

$$
\begin{gathered}
S=\dot{e}+4 e+4 \zeta \\
\dot{\zeta}=\sigma(e),
\end{gathered}
$$

and the parameter $\beta=0.008$.

Simulation results are provided in Figure 4. The four curves indicate the time response of state $x_{1}$ of the system (28) with four different controllers, respectively. It can be clearly seen that the state $x_{1}$ of controller A has steady-state error $e_{\text {ss }}=0.0135$. Meanwhile, By Lemma 3, the steady-state error of controller $\mathrm{A}$ is

$$
\lim _{t \rightarrow \infty} e=\frac{l}{c \gamma}=\frac{10}{4 \times(37 / 0.2)}=0.0135 .
$$

Thus, the simulation results of controller A are consistent with the theoretical analysis results. Similarly, the simulation results of controllers $\mathrm{B}, \mathrm{C}$, and $\mathrm{D}$ are also consistent with the theoretical analysis results (Theorem 6 and Lemma 3). Among controllers $\mathrm{B}, \mathrm{C}$, and $\mathrm{D}$, controller $\mathrm{D}$ provides the best transient performance.

The following will research the transient performance of controllers $\mathrm{B}, \mathrm{C}$, and $\mathrm{D}$ in the side of quantitative analysis. Within the boundary layer, $\dot{S}(t)=w(t)-\gamma S(t), \lim _{t \rightarrow \infty} S(t)=$ $l / \gamma$, and $e=\dot{e}=0$.

Based on the definition of sliding manifold, one has

(1) integral sliding manifold:

$$
S=\dot{e}+4 e+4 \int_{0}^{t} e d \tau
$$

If $t \rightarrow \infty$, the corresponding integral of absolute error (IAE) is

$$
\int_{0}^{t} e d \tau=S_{\mathrm{B}}-S_{\mathrm{A}}=\frac{l}{4 \gamma} .
$$

(2) Global integral sliding manifold is

$$
S=\dot{e}+4 e+4 \int_{0}^{t} e d \tau-\dot{e}(0)-4 e(0) .
$$

If $t \rightarrow \infty$, the corresponding integral of absolute error (IAE) is

$$
\begin{aligned}
\int_{0}^{t} e d \tau & =S_{\mathrm{B}}-S_{\mathrm{A}} \\
& =\frac{l}{4 \gamma}+\frac{\dot{e}(0)+4 e(0)}{4} \\
& \approx \frac{\dot{e}(0)+4 e(0)}{4} \\
& =-3.2 .
\end{aligned}
$$

(3) Nonlinear integral sliding manifold is

$$
S=\dot{e}+4 e+4 \int_{0}^{t} \sigma(e) d \tau
$$

If $t \rightarrow \infty$, the corresponding integral of absolute error (IAE) is

$$
\int_{0}^{t} \sigma(e) d \tau=S_{\mathrm{B}}-S_{\mathrm{A}}=\frac{l}{4 \gamma} .
$$




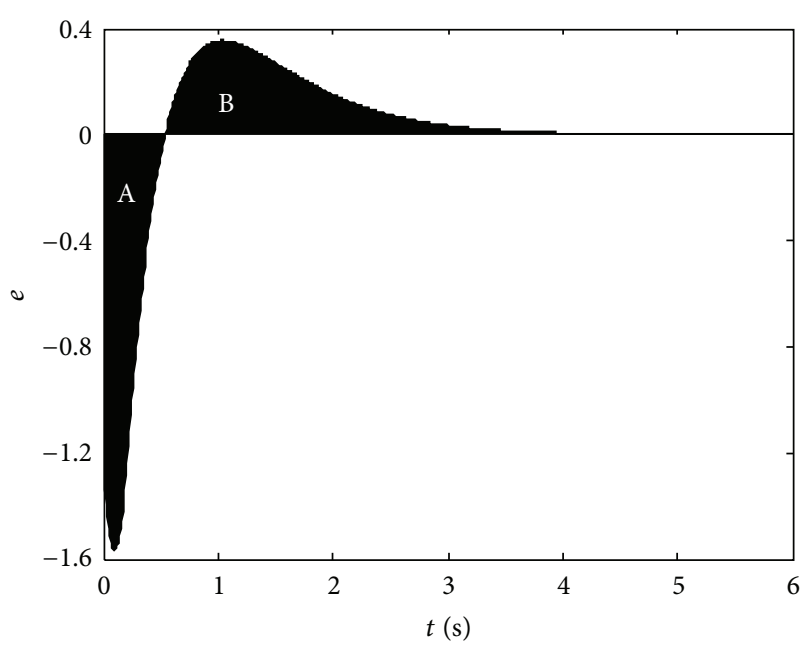

FIgURE 5: Curve of tracking error (controller B).

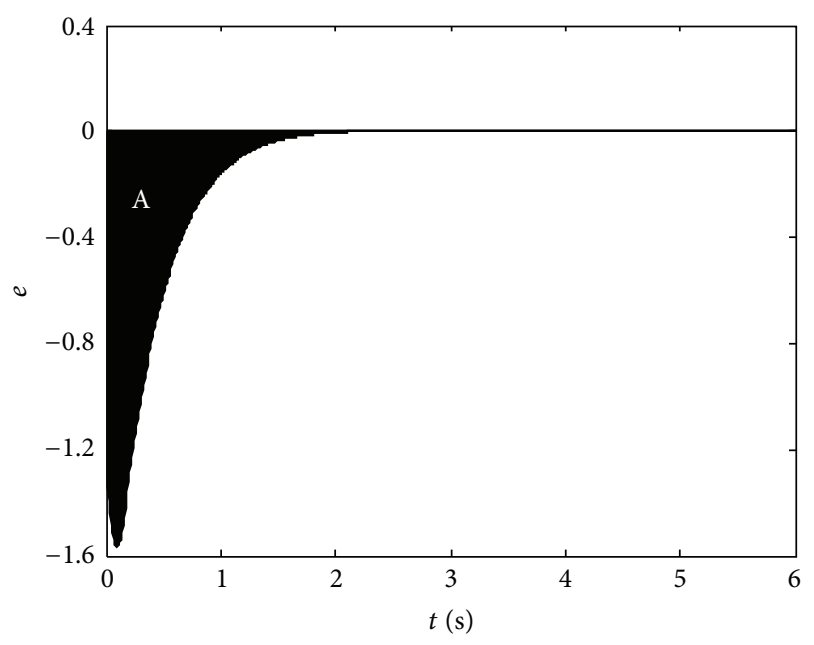

FIgure 6: Curve of tracking error (controller C).

Figures 5-7, respectively, show the tracking errors curve of controllers B, C, and D. In Figure 5, $S_{A}$ denotes the area of section $\mathrm{A}$ and $S_{\mathrm{B}}$ denotes the area of section B. Then (33) can be rewritten as

$$
S_{\mathrm{B}}=\frac{l}{4 \gamma}+S_{\mathrm{A}} \text {. }
$$

Because the boundary layer thickness $\delta$ is very little and combined with (17), the $\gamma$ usually is a very large number, it yields $S_{\mathrm{B}} \approx S_{\mathrm{A}}$. Thus, the larger the initial error $e(0)$ is, the lager the $S_{\mathrm{A}}$ and $S_{\mathrm{B}}$ are.

Figure 6 shows the tracking error curve of controller C. Although the tracking error of controller $\mathrm{C}$ has no overshoot, its rise time is very long. In Figure 6,

$$
S_{\mathrm{A}} \approx-\frac{\dot{e}(0)+4 e(0)}{4}, \quad S_{\mathrm{B}} \approx 0 .
$$

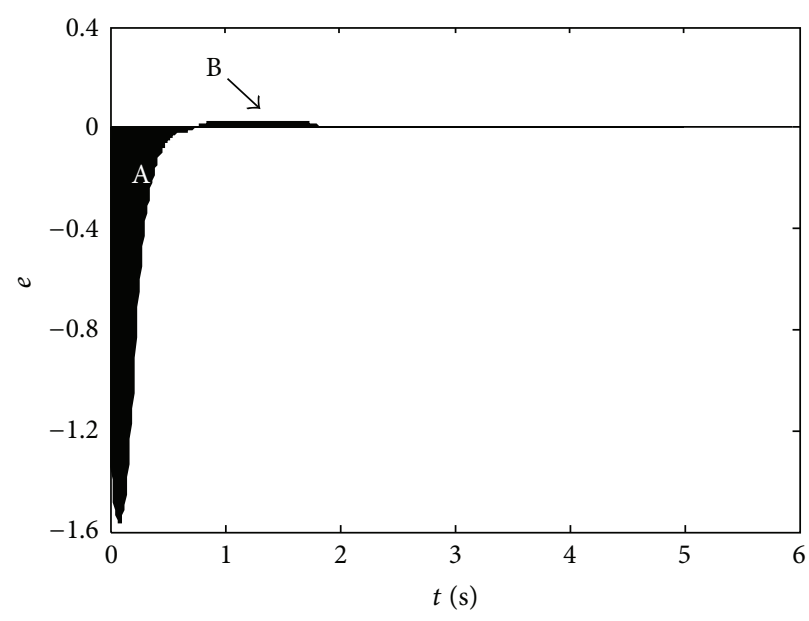

Figure 7: Curve of tracking error (controller D).

From (39), it is easily obtained that the tracking error of controller $\mathrm{C}$ is closely associated with initial conditions. If the initial error $\dot{e}(0)+4 e(0) / 4>0$, the control performance of controller $\mathrm{C}$ is even worse than controller $\mathrm{B}$.

Similarly, (37) can be rewritten as (38). Figure 7 shows the tracking error curve of controller D. Compared with controller $\mathrm{B}$, even the initial error $e(0)$ is very large, $S_{\mathrm{A}}$ is very little because of the saturation of nonlinear function $\sigma(e)$, and the $S_{\mathrm{B}}=l / 4 \gamma+S_{\mathrm{A}}$ is also very little. Therefore, the proposed control scheme can not only eliminate the steady-state error, but also avoid larger overshoot.

\section{Conclusions}

This work presents a nonlinear integral sliding mode controller design for a second order system with external disturbance. The stability and robustness to the external disturbance of the NISMC controlled system were proved by the Lyapunov stability theory and LaSalle invariance principle. Meanwhile, from the comparison of four kinds of SMC controller, we obtained that the proposed controller not only inherited the advantages of ISMC, but also provided a better transient performance. The theoretical results were confirmed through both theoretical analysis and numerical examples.

\section{Conflict of Interests}

The authors declare that there is no conflict of interests regarding the publication of this paper.

\section{Acknowledgment}

This work was partially supported by National Natural Science Foundation of China (61304239).

\section{References}

[1] C. A. Yfoulis, A. Muir, and P. E. Wellstead, "A new approach for estimating controllable and recoverable regions for systems 
with state and control constraints," International Journal of Robust and Nonlinear Control, vol. 12, no. 7, pp. 561-589, 2002.

[2] J. Wang, "Robust and nonlinear control literature survey," International Journal of Robust and Nonlinear Control, vol. 20, no. 12, pp. 1427-1430, 2010.

[3] M. Taleb, F. Plestan, and B. Bououlid, "An adaptive solution for robust control based on integral high-order sliding mode concept," International Journal of Robust and Nonlinear Control, 2014.

[4] F. Ornelas-Tellez, J. J. Rico, and R. Ruiz-Cruz, "Optimal tracking for state-dependent coefficient factorized nonlinear systems," Asian Journal of Control, vol. 16, no. 3, pp. 890-903, 2014.

[5] A. Pisano, M. R. Rapaić, Z. D. Jeličić, and E. Usai, "Sliding mode control approaches to the robust regulation of linear multivariable fractional-order dynamics," International Journal of Robust and Nonlinear Control, vol. 20, no. 18, pp. 2045-2056, 2010.

[6] Y. Shtessel, M. Taleb, and F. Plestan, "A novel adaptive-gain super-twisting sliding mode controller methodology and application," Automatica, vol. 48, no. 5, pp. 759-769, 2012.

[7] F. Plestan, Y. Shtessel, V. Brégeault, and A. Poznyak, "Adaptive sliding mode control for a class of MIMO nonlinear systems: application to an electro pneumatic actuator," Control Engineering Practice, vol. 21, no. 5, pp. 679-688, 2013.

[8] S. Mondal and C. Mahanta, "Adaptive integral higher order sliding mode controller for uncertain systems," Journal of Control Theory and Applications, vol. 11, no. 1, pp. 61-68, 2013.

[9] J.-N. Li, H. Su, Y. Zhang, Z.-G. Wu, and J. Chu, "Chattering free sliding mode control for uncertain discrete time-delay singular systems," Asian Journal of Control, vol. 15, no. 1, pp. 260-269, 2013.

[10] J.-J. E. Slotine, "Sliding controller design for systems," International Journal of Control, vol. 40, no. 2, pp. 421-434, 1984.

[11] H. Lee and V. I. Utkin, "Chattering suppression methods in sliding mode control systems," Annual Reviews in Control, vol. 31, no. 2, pp. 179-188, 2007.

[12] T.-L. Chern and Y.-C. Wu, "Design of integral variable structure controller and application to electrohydraulic velocity servosystems," IEE Proceedings D: Control Theory and Applications, vol. 138, no. 5, pp. 439-444, 1991.

[13] L. Peng and Z. Zhiqiang, "Sliding mode control approach with nonlinear integrator," Control Theory \& Applications, vol. 3, no. 28, pp. 421-427, 2011.

[14] Y. Peng, D. Vrancic, and R. Hanus, "Anti-windup, bumpless, and conditioned transfer techniques for PID controllers," IEEE Control Systems Magazine, vol. 16, no. 4, pp. 48-57, 1996.

[15] Z. Gao and X. Liao, "Integral sliding mode control for fractional-order systems with mismatched uncertainties," Nonlinear Dynamics, vol. 72, no. 1-2, pp. 27-35, 2013.

[16] D. Cho, Y. Kato, and D. Spilman, "Sliding mode and classical controllers in magnetic levitation systems," IEEE Control Systems Magazine, vol. 13, no. 1, pp. 42-48, 1993.

[17] J.-H. Lee, "Highly robust position control of BLDDSM using an improved integral variable structure systems," Automatica, vol. 42, no. 6, pp. 929-935, 2006.

[18] M. Defoort, T. Floquet, A. Kokosy, and W. Perruquetti, "A novel higher order sliding mode control scheme," Systems \& Control Letters, vol. 58, no. 2, pp. 102-108, 2009.

[19] J. J. Slotine, Application Nonlinear Control, Prentice-Hall, Upper Saddle River, NJ, USA, 1991. 

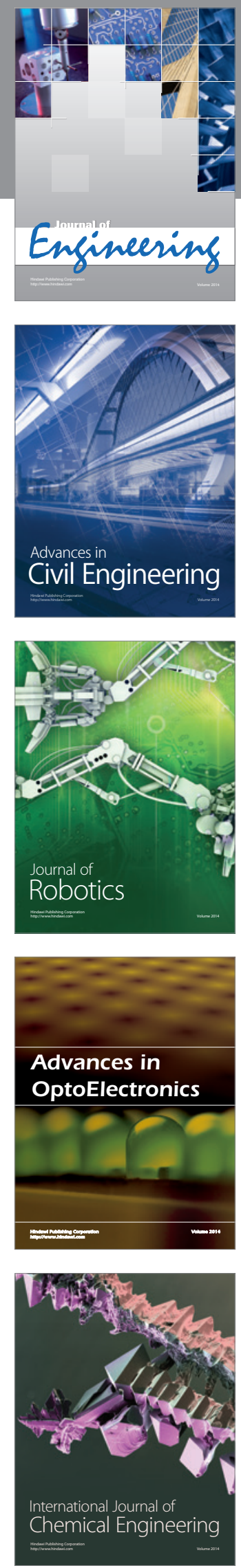

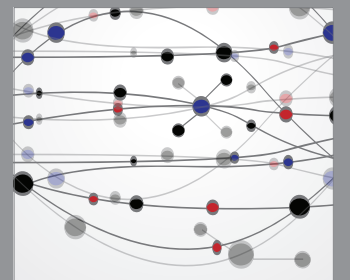

The Scientific World Journal
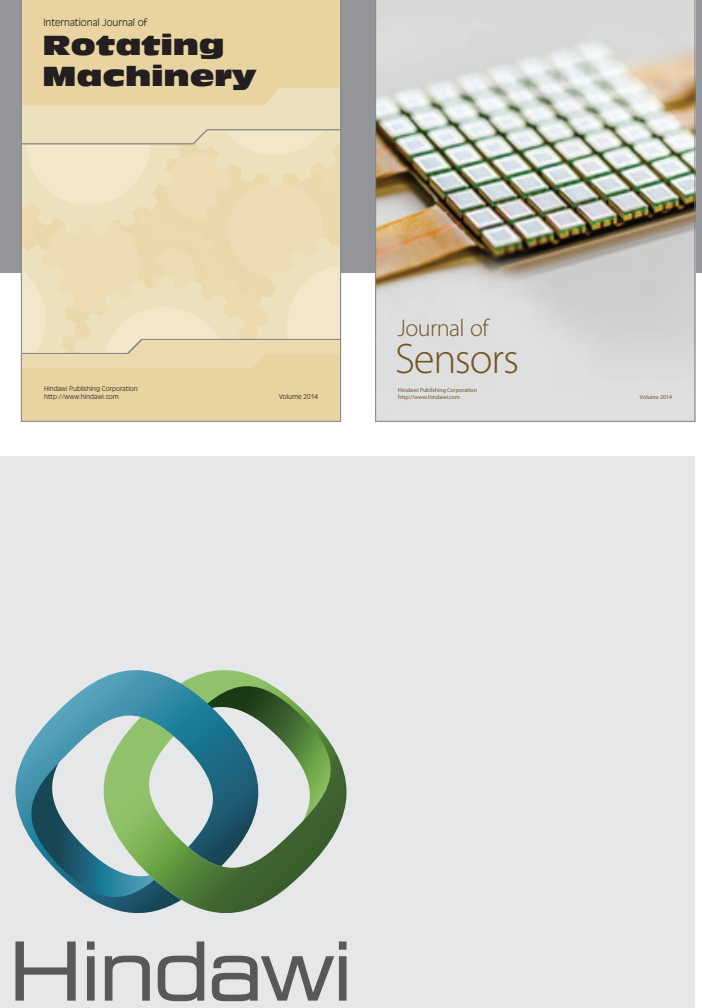

Submit your manuscripts at http://www.hindawi.com
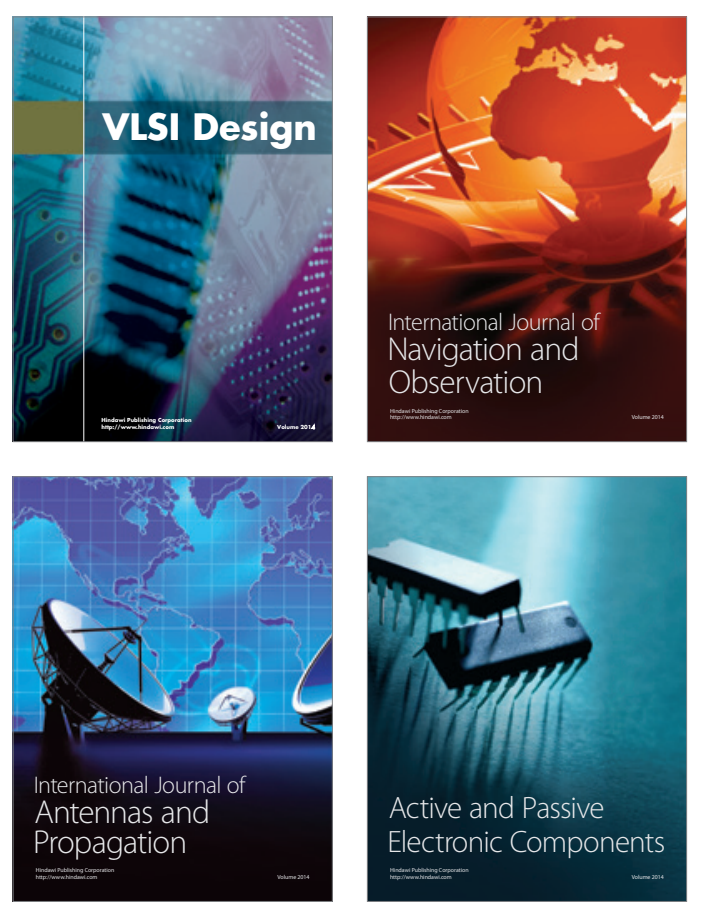
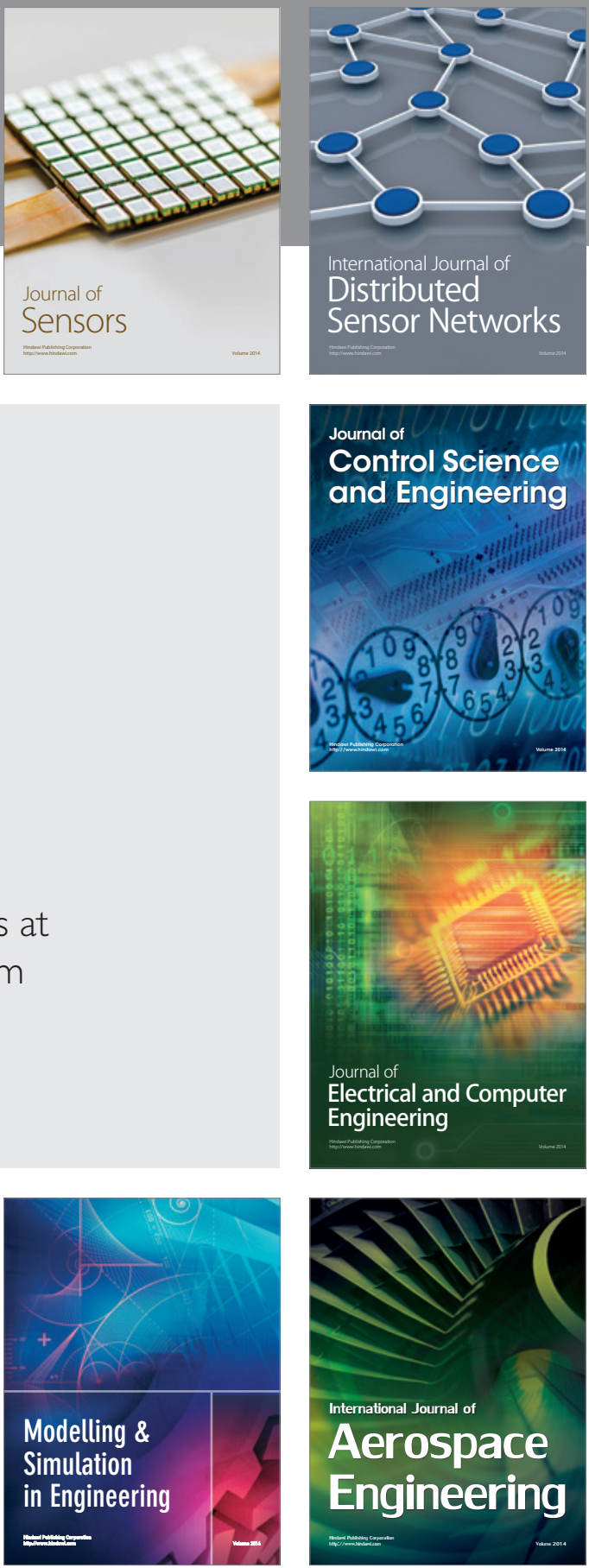

Journal of

Control Science

and Engineering
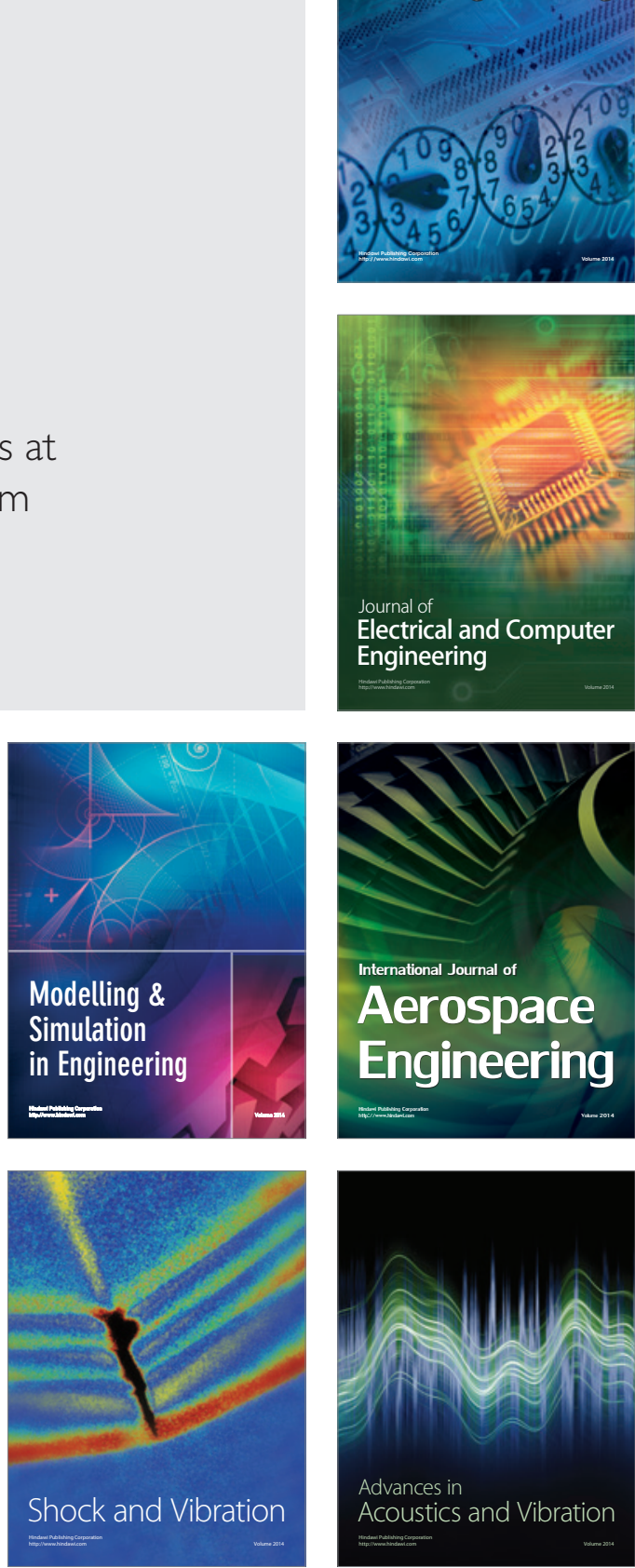